\begin{tabular}{l|l} 
REVISTA & $\begin{array}{l}\text { Revista Educación } \\
\text { ISSN: 0379-7082 } \\
\text { ISSN: 2215-2644 } \\
\text { revedu@ gmail.com } \\
\text { Universidad de Costa Rica } \\
\text { Costa Rica }\end{array}$
\end{tabular}

\title{
El trabajo colegiado y sus implicaciones: diseño de una propuesta pedagógica
}

\author{
Aguirre Gómez, Francelia Jazmín; Barraza Barraza, Laurencia \\ El trabajo colegiado y sus implicaciones: diseño de una propuesta pedagógica \\ Revista Educación, vol. 45, núm. 2, 2021 \\ Universidad de Costa Rica, Costa Rica \\ Disponible en: https://www.redalyc.org/articulo.oa?id=44066178018 \\ DOI: https://doi.org/10.15517/revedu.v45i1.42985
}

\section{(c) 1 (1)}

Esta obra está bajo una Licencia Creative Commons Atribución-NoComercial-SinDerivar 3.0 Internacional. 
Artículos científicos de experiencia social o didáctica

\section{El trabajo colegiado y sus implicaciones: diseño de una propuesta pedagógica}

Teacher Collaboration and its Ramifications: Designing a Pedagogical Approach

Francelia Jazmin Aguirre Gómez

Escuela Normal Rural "J. Guadalupe Aguilera", México

franjaz2012@gmail.com

(iD) https://orcid.org//0000-0003-2936-5161

Laurencia Barraza Barraza

Instituto Educativo GUBA, México

laura_bza@hotmail.com

(iD https://orcid.org/0000-0001-9124-7778
DOI: https://doi.org/10.15517/revedu.v45i1.42985 Redalyc: https://www.redalyc.org/articulo.oa? $\mathrm{id}=44066178018$

Recepción: 29 Julio 2020

Aprobación: 29 Octubre 2020

\section{Resumen:}

Este trabajo es un reporte parcial de una investigación en proceso, que corresponde al diseño de un taller con enfoque en aprendizaje colaborativo para resignificar el concepto y las implicaciones del trabajo colegiado en profesores de una Institución Formadora de Docentes en Durango, México. Se realizó bajo la metodología cualitativa, siguiendo el método de la Investigación-Acción, tipo práctico-deliberativo y el modelo de Elliott (1993, citado por Latorre, 2003). Para diagnosticar el problema, en la recogida de datos se utilizó la técnica de encuesta mediante la aplicación de un cuestionario de respuesta abierta a 17 docentes. Para sistematizar y validar la información se siguió el modelo de Cisterna (2005) de categorización y triangulación. Los resultados corresponden a la fase de diagnóstico y el diseño de una propuesta de intervención en función de dos categorías: conceptos e implicaciones. Como principales hallazgos se identifica que existe un sentido latente sobre el trabajo colegiado y sus implicaciones, asociado a la racionalidad técnica y competitiva. En el diseño de la propuesta se conjugan datos empíricos y teóricos, además comprende niveles de conocimiento que van desde lo epistemológico hasta lo didáctico. Se concluye que existe entre el profesorado concepciones diversificadas sobre el trabajo colegiado y sus implicaciones en el desarrollo de las actividades institucionales, que pueden ser resignificadas a partir de la instrumentación de estrategias de aprendizaje colaborativo.

Palabras CLAVE: Trabajo colegiado, Colaboración, Aprendizaje activo, Trabajo docente.

\section{Abstract:}

This article is a partial report of a study underway which focuses on collaborative learning to redefine the concept and impact of teacher collaboration among the faculty at a Teacher Training Institution in Durango, Mexico. The study used a qualitative methodology based on an Action-Research, a practical-deliberative type methodology and Elliott's model (1993, cited by Latorre, 2003). A survey technique was used to diagnose the problem and collect data through an open-ended questionnaire answered by seventeen (17) teachers. Cisterna's (2005) model of categorization and triangulation was also used to systematize and validate the information. The results were used to develop the diagnostic phase and design of an intervention based on two categories: the concept and its possible impact. According to the findings of the study, academic collaboration has been, largely, untapped and its ramifications are associated to technical issues and competition. Empirical and theoretical data were combined to design a proposal which ranges from epistemological to didactic knowledge. Based on the conclusion, since there given the many notions among teachers regarding academic teamwork and its possible impact on developing institutional activities, the concept must be redefined through the implementation of collaborative learning strategies.

KeYwords: Academic Teamwork, Teacher Collaboration, Active Learning, Teaching Practice.

\section{INTRODUCCIÓN}

Las instituciones educativas, y en particular las formadoras de docentes en Durango, México, manejan lenguajes que en apariencia son comunes y del dominio de sus integrantes; sin embargo, desde la exploración 
que se ha hecho en este sentido, se encuentra que al menos en lo que concierne al trabajo colegiado las interpretaciones son diversas, implicando un obstáculo para el desarrollo de las actividades conjuntas y mostrando el privilegio de la individualidad como práctica instituida que atomiza y fragmenta la colaboración.

El propósito de la investigación es desarrollar una propuesta de intervención pedagógica que contribuya a resignificar el concepto del trabajo colegiado y comprender sus implicaciones en la implementación para asumir conscientemente los compromisos y responsabilidades, tanto en el plano individual como institucional. Su diseño abarca cuatro etapas, la primera corresponde al planteamiento y diagnóstico del problema, la segunda al diseño de una propuesta pedagógica, la tercera a su aplicación y la última a la evaluación.

Aquí solamente se presentan los resultados de las dos primeras, debido a que las condiciones sanitarias -COVID 19- interrumpieron las actividades en los espacios escolares y no han permitido su aplicación y evaluación. No obstante, se considera que el avance de la investigación que se presenta tiene aportes no solamente en los resultados del diagnóstico, sino también en el diseño de la propuesta, porque surge de una necesidad, que a la luz de las investigaciones revisadas, atraviesa a las instituciones formadoras de docentes, por lo que puede ser aplicada en contextos similares. Asimismo, para su diseño se utilizaron modelos de planeación holística, ubicados dentro de la corriente constructivista sugeridos por Pimienta (2007). Otra característica es que en su implementación el foco es la meditación sobre las prácticas instituidas del trabajo colegiado para descubrir qué efectos están teniendo sobre la formación y el propio quehacer docente, conduciendo, de esta forma, a que el profesorado reflexione en y sobre la práctica.

El artículo se estructuró en cinco apartados: en el primero se describe el contexto en el que se realizó la investigación; el segundo corresponde al proceso de problematización, planteamiento del problema, diagnóstico del mismo, objetivos e hipótesis de acción; el tercero aborda aspectos de carácter teórico que fundamentan y soportan la propuesta, referidos al trabajo colegiado, aprendizaje colaborativo y andragogía. El cuarto describe la metodología bajo la que se desarrolló la indagación y el último presenta los resultados correspondientes al diagnóstico y al diseño de la propuesta de intervención pedagógica; para finalizar, se incluyen algunas conclusiones y recomendaciones.

\section{DESCRIPCIÓN DEL CONTEXTO}

El presente estudio se realizó en una Institución Formadora de Docentes, específicamente en una Escuela Normal Rural que opera bajo la modalidad de internado. La matrícula escolar asciende alrededor de 400 alumnos hombres, con edades que oscilan entre los 18 y 22 años, provenientes de diferentes municipios y de otros estados de la República Mexicana.

En la Escuela Normal confluyen diversidad de culturas que originan una forma peculiar de organización, política e ideológica entre los estudiantes; por su modalidad de internado, alberga principalmente jóvenes de escasos recursos, para quienes formarse como maestros representa la única posibilidad de acceder a la educación profesional, aunque no necesariamente sea su vocación.

La planta docente de la Escuela Normal está conformada por 60 personas profesoras, de las cuales 43 son de tiempo completo, algunas de ellas están comisionados, ejerciendo otras funciones fuera de la institución. Los perfiles académicos son diversos, lo que en ocasiones resulta una fortaleza para atender diferentes ámbitos de conocimiento, pero en otras se convierte en una debilidad cuando algún perfil no concuerda con los cursos de la malla curricular de los planes de estudio, lo que dificulta en gran medida la organización de la estructura académica.

Entre la formación inicial del personal docente se identifican estudios de licenciatura en educación, en otras licenciaturas e ingenierías, como se muestra en la Tabla 1. 
TABLA 1

Perfil académico

$\begin{array}{ll}\text { Perfil académico } & \text { Número de docentes } \\ \text { Licenciatura en educación } & 35 \\ \text { Otras licenciaturas } & 20 \\ \text { Ingenieria } & 5\end{array}$

Fuente: Elaboración propia.

Fuente: Elaboración propia

Respecto a la habilitación académica, la mayoría ha realizado estudios de posgrado en el área de educación, pero hasta este momento solo 8 docentes tienen grado académico de Doctorado, 24 de Maestría y 28 se quedan en nivel Licenciatura.

En el ámbito del trabajo colegiado, de manera formal e institucional se organizan las academias al inicio de cada semestre, bajo la responsabilidad de la Subdirección Académica con base en el Manual de organización del personal adscrito al subsistema de educación normal, editado por la Secretaría de Educación Pública (SEP), (1982). El documento refiere como funciones de esta subdirección "Organizar las academias de maestros conforme a los lineamientos vigentes" y "Coordinar y supervisar la integración y el funcionamiento de las academias de maestros de acuerdo con el plan, los programas de estudio y los horarios establecidos" (Secretaría de Educación Pública [SEP], 1982, p. 11). Asimismo, establece las siguientes funciones para las academias:

1. Analizar los programas de estudio con el propósito de unificar criterios para su aplicación y analizar propuestas para el desarrollo del proceso de enseñanza - aprendizaje con base en los principios establecidos en el modelo académico.

2. Proponer proyectos de docencia, investigación, extensión educativa y difusión cultural a la subdirección académica de acuerdo con la realidad educativa del plantel.

3. Determinar, aplicar y evaluar las técnicas y los recursos didácticos que apoyen el proceso de enseñanza - aprendizaje.

4. Evaluar los programas de estudio vigentes y proponer las modificaciones que proceden a la subdirección académica.

5. Proponer proyectos para la superación académica del personal docente.

6. Analizar la problemática generada en el desarrollo del proceso enseñanza - aprendizaje y proponer alternativas de solución.

7. Proporcionar información a la subdirección académica en los términos y plazos establecidos.

En el desarrollo de las actividades cotidianas de la institución, a partir de la experiencia laboral, participación y observación en reuniones colegiadas, se percibe que los documentos normativos que rigen la operación de la Escuela Normal no son del conocimiento de la mayoría del personal docente y, en algunos casos, ni por los propios directivos institucionales, por lo que el funcionamiento real de las academias dista de lo normativamente establecido. Al respecto, Covarrubias y Brito (2007) señalan que "los docentes, para sostenerse en el gremio, han desarrollado una cantidad nada despreciable de habilidades que les permiten no sólo sobrevivir, sino crear una sólida zona de confort, desconociendo incluso las normas laborales" (p. 151).

En este sentido, otro aspecto que obstaculiza el trabajo colegiado es la escasa disposición de algunos docentes por participar en las reuniones de academia, que se refleja en las constantes ausencias, escasa permanencia, incumplimiento y mínima capacidad de propuesta. Situaciones que quedan registradas en las actas de reunión con frases como: por la poca asistencia de los docentes, se decidió abordar los aspectos más relevantes para concluir en menos tiempo; la elaboración del plan de trabajo de la academia se decide retomarlo en la próxima reunión. 
Durante el desarrollo de las reuniones, se observa que la mayor participación recae en quienes presiden la academia, se adhieren al trabajo solo unos cuantos, la mayoría prestan poca atención, manifiestan inconformidades, se distraen con el celular, incumplen acuerdos, no asumen responsabilidades y atribuyen el bajo rendimiento escolar a estudiantes y directivos. En palabras de Covarrubias y Brito (2007), "en realidad, poco importa al profesor el aprendizaje de sus alumnos. Su preocupación está centrada en sus intereses personales... No está preocupado por desarrollarse académicamente él ni porque esto suceda con sus alumnos" (p. 168).

Finalmente, en el aspecto académico se percibe un ambiente laboral tenso, desconfianza entre el profesorado que complejiza las relaciones humanas y por ende el trabajo colegiado. Expresiones de crítica y descalificación son un factor constante entre los pasillos de la Escuela Normal, pues resulta más cómodo que generar propuesta y acción.

Se rescata la integración de grupos de docentes con intereses afines, quienes se organizan y trabajan por voluntad, en horarios de coincidencia, en beneficio de los estudiantes y de la institución.

\section{Proceso de PRoblematización}

Para llevar a cabo este proceso, se procedió a identificar una preocupación temática, iniciando por un listado de temas derivados de observaciones empíricas, de la experiencia laboral y el conocimiento institucional; se focalizó la atención en el trabajo colegiado.

Para continuar con la detección de las manifestaciones del tema, se utilizó la propuesta de Kemmis y McTaggart (1992) para la elaboración de una tabla aristotélica, construida a partir de "cuatro lugares comunes: enseñantes, estudiantes, tema de estudio y entorno" (p. 123).

En la tabla aristotélica se rescataron aspectos relevantes en torno al trabajo colegiado y se identificaron las diferentes perspectivas de los actores educativos. De manera institucional, se encontró que el trabajo colegiado se asocia con el funcionamiento de las academias, opera sin análisis, reflexión, gestión y seguimiento de acuerdos; requiere compromiso y disposición por parte de todos. Asimismo, se advierte mínimo conocimiento teórico y normativo al respecto, e indiferencia por parte del colectivo docente, situaciones que frecuentemente conducen al desarrollo de actividades individualizadas.

En este sentido, a partir de la tabla, se detectaron cuatro preocupaciones temáticas: a) El trabajo colegiado es prescriptivo; b) El trabajo colegiado tiene escasa aceptación entre los docentes; c) Organización y administración institucionales inadecuadas para el trabajo colegiado; d) Concepciones diversificadas sobre el trabajo colegiado y sus implicaciones. Después de valorar su pertinencia, de acuerdo con el posible grado de intervención, se decidió trabajar sobre la última preocupación.

\section{Preocupación temática}

El personal docente de la Escuela Normal tiene concepciones diversificadas sobre el trabajo colegiado y sus implicaciones.

\section{Diagnóstico}

Para corroborar la problemática, se diseñó un cuestionario de respuesta abierta; se aplicó a 17 docentes. Al sistematizar la información, aparecieron las categorías: concepto, utilidad, organización institucional, cultura laboral, valores y actitudes. El diagnóstico reveló: 
1. Existen diferentes conceptos respecto al significado del trabajo colegiado: acción, actividad, situación estratégica, compartir vivencias, gestión y decisión.

2. Se percibe la utilidad del trabajo colegiado para planear, diseñar proyectos, analizar la práctica docente, mejorar institucionalmente.

3. La organización institucional escasamente favorece el trabajo colegiado: no hay coincidencia en horarios, escasez de espacios e insuficiente planeación para su desarrollo.

4. En relación con la cultura laboral, señalan que hay resistencias al cambio, exigua participación e involucramiento en las actividades conjuntas, inercias que impiden el juicio crítico.

5. El trabajo colegiado exige responsabilidad, compromiso, empatía, reconocimiento, respeto; sin embargo, en la práctica se evidencia una carencia actitudinal.

En general, podría concluirse que existe en el profesorado información diversificada sobre el trabajo colegiado y las implicaciones que presenta para los colectivos docentes y las instituciones. También es un término de uso común y cotidiano en los espacios académicos, pero no siempre tiene el mismo significado para la comunidad educativa.

Con base en la información recabada, se consideró importante que el personal docente utilice un lenguaje común sobre el tema, para agilizar los procedimientos institucionales y la toma de acuerdos. Cuando un colectivo no maneja códigos compartidos, se corre el riesgo de tergiversar expresiones, propiciar malos entendidos que desestabilizan y obstaculizan el desarrollo de las actividades académicas e institucionales. En este sentido, se pensó que un primer elemento a considerar en esta propuesta es la construcción de un concepto compartido del término trabajo colegiado. Para tal efecto se planteó la siguiente interrogante: ¿Cómo resignificar las concepciones del profesorado de la Escuela Normal sobre el trabajo colegiado y sus implicaciones?

\section{Objetivo}

Diseñar un taller con enfoque en aprendizaje colaborativo para resignificar el concepto y las implicaciones del trabajo colegiado en el profesorado de una Institución Formadora de Docentes en Durango, México.

\section{Hipótesis de acción}

Resignificar el concepto y las implicaciones del trabajo colegiado en el profesorado de una Institución Formadora de Docentes en Durango, México, mediante el diseño e implementación de un taller con enfoque en aprendizaje colaborativo.

\section{Trabajo colegiado}

Entre los autores y autoras que han abordado el tema del trabajo colegiado se encuentran: Barraza y Guzmán (2007), Barraza (2012), Esteva (2013), Barraza y Barraza (2014), Martínez, Ruiz, Galindo y Galindo (2015), Rodríguez y Barraza (2015), Landín y Sánchez (2016), Hernández, Carro y Carrasco (2016), Quintero y Romero (2017), Becerra-Chávez y Hernández-Hernández (2018), Martínez, Ramírez y Ramírez (2019). Las investigaciones citadas en su mayoría se ubican en el paradigma interpretativo y atraviesan los niveles educativos de básica, media superior y superior, cinco de estas se realizaron en Instituciones Formadoras de Docentes y están asociadas a mejorar la enseñanza, el aprendizaje y la práctica docente. Algunos elementos comunes que se reportan son: la apreciación del trabajo colegiado desde un enfoque pragmático, instituido desde la prescripción y la norma. La presencia de una cultura docente e institucional no favorecedora para la 
colegialidad. El esfuerzo de grupos al interior de las instituciones por instituir el trabajo colegiado como una práctica y/o espacio de reflexión, auto-observación y autocrítica que contribuya a la redefinición del trabajo académico e impulse procesos de mejora. Se identifica la ética como un componente que limita o potencia las actividades conjuntas. Se destacan como categorías de análisis colegialidad científica (Hernández, Carro y Carrasco, 2016), colegialidad competitiva (Barraza y Barraza, 2014), colegialidad obligada y colegialidad artificial (Barraza y Guzmán, 2007).

La literatura muestra diversos conceptos sobre el término trabajo colegiado, así Barraza y Guzmán (2007) conciben al trabajo colegiado como una estrategia impulsada desde la política educativa, que tiene como objetivo apoyar a los colectivos docentes para que transiten de una cultura individualista a una colaborativa, con la finalidad de mejorar la práctica docente y los procesos de organización y administración institucionales. Para Esteva (2013) es un medio para articular las actividades referidas tanto a la enseñanza como el aprendizaje y donde la meta es la formación integral de los estudiantes. Martínez, et al., (2015) lo conciben como un espacio de interacción entre docentes donde se dialoga, problematiza, reflexiona y se colabora en la solución de temáticas asociadas a la enseñanza, el aprendizaje y la función docente. Becerra-Chávez y Hernández-Hernández (2018) asumen el trabajo colegiado como espacio de reunión de los actores para reflexionar sobre la práctica docente y generar propuestas que apoyen en la resolución de problemáticas relacionadas con la enseñanza y el aprendizaje. Martínez, et al., (2019) plantean que es un medio fundamental para compartir experiencias y conocimientos, a través del cual se puede lograr una mejor formación integral de las y los estudiantes y de la calidad educativa.

Los conceptos tienen en común la reflexión, el diálogo, el intercambio y la colaboración. La meta es la mejora, el cambio o la transformación de las culturas institucionales, la práctica docente, la enseñanza y el aprendizaje. De esta forma, el trabajo colegiado se convierte en la estrategia, el medio y/o el espacio para establecer procesos institucionales que contribuyan a impulsar culturas ancladas en la colaboración y el compromiso de los actores presentes en los centros escolares.

Las características que Barraza (2012) le atribuye a este tipo de trabajo son: se realiza entre colegas, entre pares, implica relaciones de horizontalidad, centrado en el liderazgo académico, requiere credibilidad, reconocimiento, participación activa, diálogo, pensamiento flexible y abierto, disposición para la escucha y alto grado de compromiso, tolerancia, paciencia, respeto y corresponsabilidad. Demanda cambios, tanto en la organización como en la cultura institucional.

\section{Aprendizaje colaborativo}

La hipótesis de acción que guía esta propuesta contempla el aprendizaje colaborativo como el enfoque del taller, mediante el cual se pretende resignificar el concepto y las implicaciones del trabajo colegiado. Al respecto Barkley, Cross y Howell (2012) señalan que "el aprendizaje colaborativo alude a las actividades de aprendizaje expresamente diseñadas para parejas o pequeños grupos interactivos y realizadas por ellos" ( $p$. 17). Le atribuyen las siguientes características: tiene un diseño intencional, usa la colaboración, debe haber una enseñanza significativa y se usa principalmente en la educación superior. Tiene su base epistemológica en el constructivismo social. Matthews (1996, p. 101, en Barkley, et al., 2012) indica que

"El aprendizaje colaborativo se produce cuando los alumnos y profesores trabajan juntos para crear el saber (...) Es una pedagogía que parte de la base de que las personas crean significados juntas y que el proceso las enriquece y las hace crecer" (p. 19).

Barkley, et al., (2012) señalan que "todos los métodos de aprendizaje colaborativo hacen hincapié en la importancia de la interacción promotora y de la responsabilidad individual” (p. 21). La primera consiste en que los alumnos se apoyen entre sí y la segunda se refiere a que el grupo es responsable de alcanzar los objetivos y cada uno se compromete a realizar la tarea que le corresponde. 
Díaz-Barriga y Hernández (2010) señalan que el foco de análisis de la perspectiva colaboracionista es la actividad conjunta, y los mecanismos mediante los cuales se pasa a formar parte de una comunidad de práctica, la que consiste en un conjunto de personas que comparten intereses comunes respecto a un tema o problema y profundizan en su conocimiento para afrontarla y exige un compromiso mutuo.

Roselli (2016) plantea que la colaboración es un proceso colectivo, donde todos los integrantes del grupo o equipo intervienen conjuntamente en la realización de la tarea y en el aprendizaje colaborativo el conocimiento se entiende como un "proceso de negociación o reconstrucción conjunta de significados" (p. 224).

Lillo (2013) advierte que el aprendizaje colaborativo no se presenta de forma natural al otorgarles una instrucción a los estudiantes, sino que debe haber intencionalidad. Asimismo, plantea algunas dificultades para su desarrollo, tales como: el entendimiento entre los miembros del grupo, el abuso de unos sobre el esfuerzo de otros y el reconocimiento de la colaboración como una estrategia válida para la generación de aprendizajes. En este sentido, demanda la revisión del número de estudiantes por grupo, el cambio de rol del profesor y la disposición organizativa de las aulas e instituciones.

La perspectiva colaborativa toma el supuesto de la teoría vigotskiana del aprendizaje entre pares y la mediación como herramientas para la construcción del conocimiento. En esta concepción se trata de evitar la dependencia que pudiera tener el alumnado del docente, se pretende que este se convierta en un acompañante o mediador. Revelo-Sánchez, Collazos-Ordoñez y Jiménez-Toledo (2018) sobre el aprendizaje colaborativo señalan que se sustenta en enfoques cognitivos, donde el centro es el estudiante y sus procesos de aprender y se promueve el aprendizaje significativo. Los resultados son tan importantes como los procedimientos y las rutas para la consecución de nuevos aprendizajes.

Se ha optado por el aprendizaje colaborativo porque sus características son propicias para la implementación de la propuesta, al tener su fundamento en la teoría sociocultural ofrece la posibilidad de construcción colectiva del conocimiento; permite el aprendizaje entre pares, situación que otorga relaciones de horizontalidad que posibilita la interacción en situaciones más igualitarias, los grupos de trabajo son pequeños, el papel protagónico es del alumnado, el docente aparece como el mediador que apoya, acerca y facilita el acceso al conocimiento. Existe una interdependencia positiva donde la responsabilidad del aprendizaje es compartida.

\section{Perspectiva andragógica del aprendizaje}

Para complementar los referentes teóricos y debido a que la intervención se realizará con personas adultas, se plantean algunas características del aprendizaje desde la perspectiva andragógica, las metodologías de enseñanza y aprendizaje deben ser participativas y de autogestión.

Piña, Rodríguez y Rodríguez (2016) indican que la experiencia del estudiante, el interés por el objeto a estudiar y las relaciones de horizontalidad son fundamentales en este tipo de aprendizaje. Plantean tres principios nodales: 1) horizontalidad, 2) participación y 3) autogestión; el primero refiere a las relaciones entre profesor y estudiantes, los cuales pueden intercambiar roles, el nivel de compromiso es el mismo para el facilitador que para el estudiante. Significa que la autoridad la concede el conocimiento específico sobre el tema.

Según Adams (1990, en Piña, et al., 2016), la participación "es el acto de compartir algo, es un dar y recibir, involucrarse en un proyecto común" (p.16), tiene un componente crítico, por lo tanto, estimula el razonamiento, el análisis, la argumentación y la capacidad de propuesta. En tanto que la autogestión se refiere a la capacidad de autorregulación y se vincula a la evaluación como proceso autodirigido que permite al estudiante racionalizar los aprendizajes significativos para satisfacer sus necesidades e intereses. 


\section{MÉTOdo}

El trabajo que se presenta corresponde al diseño de una propuesta de intervención pedagógica que se derivó del diagnóstico en torno al trabajo colegiado en una Institución Formadora de Docentes (IFD) del estado de Durango, México. El objetivo fue diseñar un taller con enfoque en aprendizaje colaborativo para resignificar este concepto y sus implicaciones. Se realizó bajo la metodología cualitativa, considerando que es inductiva, holística, se desarrolla en un escenario natural y los investigadores están implicados (Sandín, 2003). Se utilizó el método de la Investigación-Acción (I-A), porque permite el análisis y la reflexión conjunta sobre la práctica docente y ofrece la posibilidad de intervenir para solucionar, cambiar o transformar. Latorre (2003), lo define como "una indagación práctica realizada por el profesorado, de forma colaborativa, con la finalidad de mejorar su práctica educativa a través de ciclos de acción y reflexión” (p. 25).

Elliot (1993, citado por Latorre, 2003) refiere que algunas de las características de la I-A, son: supone la reflexión simultánea sobre los medios y los fines, participativa, reflexiva e integra teoría y práctica. Para efectos de esta investigación se eligió el modelo de este autor y se ubicó en el tipo práctico -deliberativo.

El criterio para la elección de los 17 sujetos participantes fue la disposición a colaborar, debido a que esta metodología exige el acuerdo de los participantes para poder generar procesos de reflexión, que redunden en la mejora, el cambio y/o la transformación. Para sistematizar y validar la información se siguió el modelo de Cisterna (2005) de categorización y triangulación.

El procedimiento que se siguió fue:

1. Mediante la tabla aristotélica se identificaron algunas preocupaciones temáticas:

- El trabajo colegiado en la Escuela Normal es prescriptivo.

- Desconocimiento de los documentos normativos que rigen el funcionamiento de las academias.

- El trabajo colegiado tiene escasa aceptación por parte de la mayoría del personal docente.

- La escasa confianza entre docentes complejiza el trabajo colegiado.

- Concepciones diversificadas sobre el trabajo colegiado y sus implicaciones.

- Los fines institucionales no son compartidos por la mayoría del personal docente.

2. Se analizaron las preocupaciones temáticas y se eligió en función del posible grado de intervención, que es la que se desarrolla en esta investigación.

3. Se utilizó la técnica de encuesta mediante la aplicación de un cuestionario de respuesta abierta, para conocer las concepciones del profesorado sobre el trabajo colegiado y sus implicaciones.

4. Se sistematizó la información como se muestra en la Tabla 2, usando la estrategia de categorización y triangulación.

5. Se analizó la información a partir de las categorías definidas, se llevó a cabo una triangulación teórica y de datos para identificar elementos comunes y divergentes en la literatura revisada y entre los datos empíricos aportados por los informantes. Al final, se hizo una confrontación entre los resultados de la investigación con los aportes teóricos.

6. Se redactó el informe correspondiente al diagnóstico y se diseñó una propuesta de intervención pedagógica, consistente en un taller y la planeación didáctica por sesión, que aparece en los resultados. 


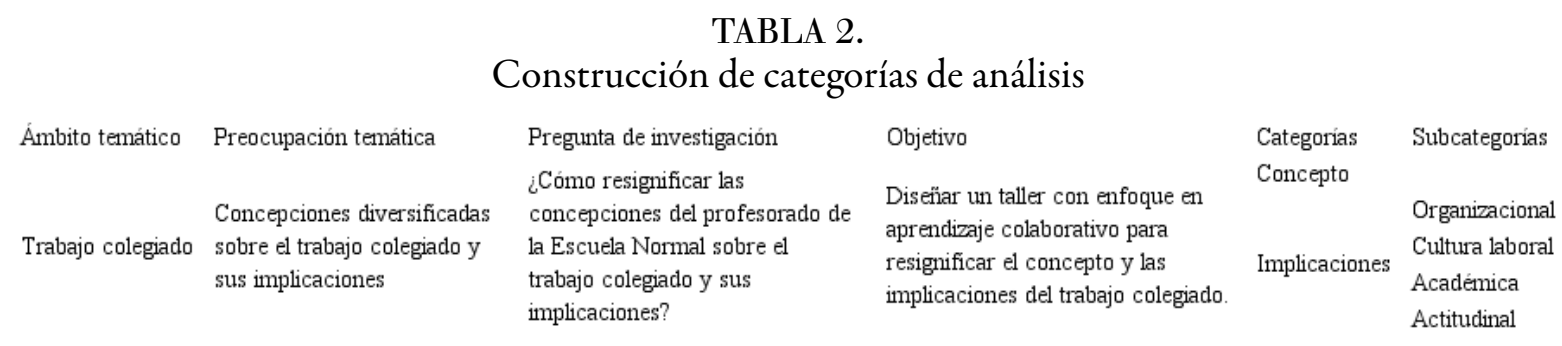

Fuente: Elaborada a partir del modelo de Cisterna (2005).

\section{Resultados}

La Investigación-Acción plantea por lo menos dos momentos para recoger información: diagnóstico e intervención. Los resultados que se presentan en este reporte son los que corresponden a la aplicación del instrumento para diagnosticar el problema y el diseño de una propuesta pedagógica que se concreta en un taller dirigido a profesores de una institución formadora de docentes, con el objetivo de resignificar el concepto y las implicaciones del trabajo colegiado, para favorecer una visión común en la toma de decisiones y cumplimiento de acuerdos institucionales.

Para analizar la información, se definieron dos categorías: concepto e implicaciones. En la categoría concepto se encontró que el trabajo colegiado se asocia al trabajo de academia, a las acciones, actividades, estrategias, gestiones y decisiones que se hacen en conjunto y entre iguales; también se considera un medio para buscar rutas o caminos comunes, un espacio de intercambio, confrontación de ideas y compartir experiencias; asimismo, hay quien lo concibe como la columna vertebral de los procesos educativos. Perciben su impacto en la enseñanza, el aprendizaje, la planeación y evaluación; lo consideran útil para alcanzar fines, construir y consolidar proyectos comunes.

Algunas de las siguientes respuestas, recuperadas en el instrumento, muestran la diversidad de concepciones y los aspectos en común que asume el profesorado:

- Las acciones realizadas en conjunto por parte de cada uno de los individuos pertenecientes a la planta docente. (I3)

- El trabajo colegiado al interior de las academias permite organizar y sistematizar los procesos educativos, gestionar y planificar. (I11)

- Trabajo en conjunto de los docentes de cada institución con el fin de mejorar la práctica docente y los procesos de gestión escolar, institucional, también cabe mencionar que es la columna vertebral de los procesos educativos, gestiones y decisiones en conjunto. (I15)

- Compartir las vivencias y experiencias de los docentes y trabajadores con la finalidad de potenciar las capacidades de los alumnos y/o institución. (I17)

Los aspectos comunes que se perciben en las respuestas son: es un trabajo que se hace en conjunto, grupo y/ o colectivo; se realiza en colaboración y tiene aplicación en diferentes áreas o momentos; es un trabajo dirigido y normado institucionalmente donde se privilegia la experiencia sobre el conocimiento. En los aspectos divergentes se identifica que el trabajo colegiado es un soporte que se desarrolla de manera integral, tiene una relación de horizontalidad y es el conocimiento el que otorga el liderazgo académico.

La categoría implicaciones se refiere a la participación, el compromiso y el reconocimiento de aquellas condiciones que son indispensables para desarrollar el trabajo colegiado. A continuación, se plantean algunas respuestas que las ejemplifican: 
- Que no se coincida en horarios, que no haya un espacio designado para las reuniones, que no se lleven acuerdos entre los participantes y que no se involucren y participen de la misma manera. (I7)

- La resistencia al cambio. (I11)

- Mejorar la práctica docente, ocasionalmente considero que no se aprovecha el tiempo en dichas reuniones, un espacio para compartir y reflexionar sobre mis debilidades y fortalezas con el fin de mejorar la docencia. (I15)

- Compromiso, tolerancia, compartir, reconocer debilidades, voluntad. (I16)

Aquí se identifican cuatro subcategorías: organizacional, cultura laboral, académica y actitudinal. A la primera se vincula el tiempo, traducido en horarios, puntualidad, asistencia, planeación y uso eficiente de los recursos. El trabajo colegiado implica coincidir en tiempos, por lo que desde la organización escolar debe preverse, para evitar la impuntualidad y las ausencias, además requiere de un espacio y de personal asignado.

En la cultura laboral se enfatiza la resistencia al cambio, el incumplimiento de acuerdos, escasa o forzada participación, mínimo involucramiento en las actividades que demandan de este tipo de trabajo. En lo académico las implicaciones se inscriben en la mejora institucional y de práctica docente, asociada a la creación de proyectos y materiales educativos. En lo actitudinal, implica despojarse del egoísmo, ser empático, respetuoso, responsable, comprometido y tolerante.

Los resultados muestran que el trabajo colegiado, desde la percepción docente, debe ser normado, regulado, estructurado y jerárquico, instrumentado desde los espacios académico-administrativo; por lo que puede afirmarse que sigue inscrito dentro de la prescripción y todavía no alcanza la madurez para considerarlo como una práctica instituida, coincidiendo con Barraza y Guzmán (2007), quienes sostienen que en las Instituciones Formadoras de Docentes existe una colegialidad artificial.

Entre los obstáculos que se advierten, se perciben procesos inerciales que conllevan a la escasa participación, a la resistencia, a la asunción de una postura crítica y con sentido, sin aportaciones significativas y más orientados a un cumplimiento prescrito. En congruencia con Esteva (2013), quien muestra los esfuerzos de un equipo docente por instituir esta práctica; sin embargo, advierte un conjunto de resistencias que la obstaculizan.

En este primer acercamiento al concepto, el profesorado no percibe al trabajo colegiado como un espacio de aprendizaje, orienta sus definiciones hacia lo pragmático y la racionalidad técnica, es el medio para alcanzar el fin. Este podría ser un "significado latente" (Quintero y Romero, 2017, p. 36) que proyecta un tipo de cultura institucional e identidad del colectivo docente. Podría significar que se unen para buscar estrategias específicas que apoyen en la solución de problemas comunes utilizando criterios prácticos y más cercanos a la experiencia.

En la subcategoría actitudinal aparece la expresión despojarse de egoísmos, que podría significar: 1) El trabajo colegiado se inscribe en una colegialidad competitiva (Barraza y Barraza, 2014), obligada y artificial (Barraza y Guzmán, 2007) en donde "La gestión se ha convertido en la asignación de recursos para los docentes" (Quintero y Romero, 2017, p. 40); 2) El colectivo docente trabaja en colaboración buscando el beneficio personal. Desde esta postura, el trabajo se realiza desde la desconfianza, por lo tanto, se evita la aportación de ideas en el temor de que otros se apropien de ellas y las utilicen para la obtención de estímulos económicos (Barraza, 2012). Estos supuestos dan cuenta de las bondades que presenta el trabajo colegiado, pero también hacen notorias sus dificultades y los marcos desde donde se intenta discutirlo e instituirlo en los centros educativos.

Derivado de los resultados del diagnóstico, siguiendo el esquema de la Investigación-Acción se procedió a la construcción de una propuesta pedagógica, consiste en el diseño del taller denominado: El trabajo colegiado y sus implicaciones, dirigido a docentes y directivos de la Escuela Normal, el cual que se detalla en el Anexo 1 y se concreta en las planeaciones didácticas correspondientes, presentadas en el Anexo 2 y Anexo 3. 
El diseño del taller se realizó a partir de los indicadores propuestos por la Secretaría de Educación del Estado de Durango (SEED) (2019) para la estructuración de programas de actualización y capacitación. Las planeaciones didácticas se elaboraron a partir de una de las propuestas de Pimienta (2007).

Considerando que el trabajo colegiado es una línea de política educativa para la Educación Normal, se pretende que, a partir de la colaboración de profesores, puedan desarrollarse estrategias que influyan en la mejora institucional.

La estructura del Taller contempla la presentación y justificación, donde se describen el contexto de aplicación, el origen y la viabilidad de la propuesta; se definen el propósito del taller, perfil de ingreso y egreso de los docentes participantes, así como las competencias que se pretenden favorecer; se establecen contenidos curriculares por unidad de aprendizaje y el número de sesiones necesarias para su desarrollo; se detalla el programa curricular, estableciendo mecanismos de evaluación y las horas teóricas y prácticas requeridas para cada una de las sesiones; se establece el cronograma de actividades, se explicita la metodología de trabajo, las estrategias didácticas y los criterios de evaluación de procesos y productos, así como el nivel de logro; además, se plantean las normas de acreditación y materiales de trabajo.

Para concretar la propuesta se diseñaron planeaciones didácticas por sesión, considerando los elementos de la metodología constructivista: número de clase, tema, nivel de asimilación, objetivos de aprendizaje, título de la clase, método, estrategias de enseñanza-aprendizaje, recursos, reactivación de conocimientos previos, situación problemática, aplicación de conocimientos, construcción de significados, organización del conocimiento, evaluación del proceso, tarea y materiales de trabajo (Pimienta, 2007).

Siguiendo la lógica de la I-A, esta planeación corresponde a un primer ciclo de intervención, por lo que, a partir de los resultados de su implementación, podrán replantearse nuevas formas de actuación en beneficio del trabajo colegiado de la institución.

\section{Conclusiones}

El trabajo colegiado es un término que presenta una diversidad de concepciones e interpretaciones, se le asignan múltiples funciones y usos, frecuentemente aparece ligado al trabajo colaborativo por lo que las fronteras entre un concepto y el otro tienden a diluirse; sin embargo, para efectos institucionales, resulta factible el manejo de códigos y lenguajes comunes que les permitan a los colectivos docentes establecer canales de comunicación en frecuencias sintonizadas, que favorezcan la toma de acuerdos y decisiones en marcos que delimiten el alcance de esto, a fin de identificar las implicaciones que plantean en lo individual e institucional.

Se puede afirmar que la concepción docente sobre el trabajo colegiado se vincula a la colegialidad artificial y competitiva, se ubica dentro de una racionalidad técnica al considerarlo normado, regulado, jerarquizado y propuesto desde los órganos académico-administrativos. Se identifica la existencia de culturas institucionales centradas en la individualidad, donde las actividades conjuntas son apreciadas como algo que implica esfuerzo y tiempo adicional.

Se advierten esfuerzos por instituir el trabajo colegiado como un soporte institucional que atraviese y vincule integralmente las prácticas, fomente relaciones más horizontales, impulse el liderazgo académico y se convierta en la columna vertebral de los procesos educativos.

Los resultados muestran que el profesorado es consciente de las demandas del trabajo colegiado, las expresan por lo menos en dos ámbitos: institucional y personal. La primera referida a la organización y cultura laboral, la segunda a la cuestión académica y ética. Asimismo, perciben como principales obstáculos la individualización y competencia entre docentes.

En el diseño de la propuesta pedagógica, que se concreta en el taller El trabajo colegiado y sus implicaciones, se conjugan datos empíricos y teóricos, los niveles de conocimiento que se involucran van desde lo epistemológico hasta lo didáctico. Se realizó con enfoque en aprendizaje colaborativo, considerando que 
requiere del trabajo conjunto, aprendizaje e interacción entre pares, es intencional, tiene su base en el constructivismo social, promueve la responsabilidad y el apoyo mutuo para alcanzar metas comunes.

La propuesta se diseñó para que el profesorado construya significados a la luz de revisar concepciones, analizar prácticas y reflexionar sobre sus acciones y cómo están aportando al desarrollo del quehacer institucional y hasta qué punto lo están limitando y obstaculizando. Se espera que en la implementación de la propuesta el trabajo colegiado posea códigos compartidos y se convierta en una estrategia que vertebre las actividades institucionales.

\section{Recomendaciones}

Colegialidad competitiva es un concepto que en primera instancia podría parecer hasta contradictorio; sin embargo, muestra la concepción utilitarista del trabajo colegiado, significa que se entrelazan intereses académicos y económicos, donde los primeros podrían asociarse a la colectividad y los segundos a la individualidad; desde este escenario, la colegialidad se estaría desarrollando desde la desconfianza y escasa credibilidad. Se considera que para futuras investigaciones este podría ser explorado con mayor profundidad.

El método de la investigación-acción representa una oportunidad para impulsar procesos de transformación. Desde una perspectiva crítica permite revisar prácticas y procesos instituidos, a fin de generar propuestas de intervención que contribuyan a repensar el trabajo académico y de investigación que se realiza en las instituciones de educación superior.

\section{REFERENCIAS}

Ander-Egg, E. (1981). Metodología y práctica de la animación socio-cultural. España: Marsiega, D.L.

Barkley, E. Cross, K. y Howell, C. (2012). Técnicas de aprendizaje Colaborativo. (2ª ed.). España: Morata.

Barraza, L. y Guzmán, A. (2007). El trabajo colegiado en las Instituciones Formadoras de Docentes. México: COMIE. Recuperado de https://bit.ly/3bIFjES

Barraza, L. (2012). El trabajo colegiado una estrategia de mejora para la práctica docente. En M. Navarro y O. González (Coords.), La gestión de los Centros educativos (pp. 126-140). Durango, México: REDIE. Recuperado de http:/ /iunaes.mx/wp-content/uploads/2013/04/coleccionlibro5.pdf

Barraza, L. y Barraza, I. (2014). El colegiado y sus realidades. Revista Ra Ximhai, 10(5), 467-479. Recuperado de htt ps://www.redalyc.org/pdf/461/46132134029.pdf

Becerra-Chávez, A. y Hernández-Hernández, F. (2018). Experiencia de trabajo colegiado en ciencias básicas en la Universidad Politécnica de Querétaro. Revista de Pedagogía Crítica, 2(5), 27-33. Recuperado de https://bit.ly /30Falr2

Cisterna, F. (2005). Categorización y triangulación como proceso de validación del conocimiento en investigación cualitativa. Theoria, 14(1), 61-71. Recuperado de https://www.redalyc.org/pdf/299/29900107.pdf

Covarrubias, F. y Brito O. (2007). Una pálida sombra: la identidad del profesor mexicano. Colección mástextos, 28. México: UPN. Recuperado de http://200.23.113.59:8080/handle123456789/922

Díaz-Barriga, F. y Hernández, G. (2010). Estrategias docentes para un aprendizaje significativo (3a ed.). México: Mc Graw-Hill.

Esteva, A. (2013). El trabajo colegiado docente normalista: entre lo instituido y lo instituyente. Reflexiones a partir de un estudio de caso. En P. Ducoing Watty, (Coord.), La Escuela Normal: una mirada desde el otro (pp. 355-388). México: iisue-unam. Recuperado de https://issuu.com/educomplexus/docs/la_escuela_normal._una_mirada_ desde

Hernández, F., Carro, A. y Carrasco, M. (2016). El trabajo colegiado en la investigación: El Cuerpo Académico Gestión y Políticas Educativas de la Universidad Autónoma de Tlaxcala. Debates en Evaluación y Currículum/Congreso 
Internacional de Educación Evaluación, 2(2), 3997-4008. Recuperado de https://posgradoeducacionuatx.org/p df2016/F029.pdf

Johnson, D., Johnson R. y Hulobec, E. (1999). El aprendizaje cooperativo en el aula. Buenos Aires: Paidós. Recuperado de http://conexiones.dgire.unam.mx/wp-content/uploads/2017/09/El-aprendizaje-cooperativo-en-el-aula-Jo hnsons-and-Johnson.pdf

Kemmis, E. y McTaggart, R. (1992). Cómo Planificar la Investigación Acción. (1 ${ }^{a}$ Reim.). Barcelona, España: Latres.

Landín, M. y Sánchez, S. (2016). El trabajo colegiado, un espacio para narrar las experiencias desde la práctica docente. Entreciencias: Diálogos en la Sociedad del Conocimiento, 4(11), 1-18. Recuperado de http://www.redalyc.org/a rticulo.oa?id $=457647810002$

Latorre, A. (2003). La investigación-acción. Conocer y cambiar la práctica educativa (1ª ed.). España: Graó.

Lillo, F. (2013). Aprendizaje Colaborativo en la Formación Universitaria de Pregrado. Revista de Psicología, 2(5), 109-142. Recuperado de http://sitios.uvm.cl/revistapsicologia/revista-detalle.php/4/25/resumen-

Martínez, J., Ramírez, A. y Ramírez, J. (2019). Experiencias del trabajo colegiado en la Universidad Autónoma de Nayarit: una mirada desde las academias. Revista Digital de Investigación Turística. KIKAME, 7(7), 55-70. Recuperado de https://www.researchgate.net/publication/338594655_

Martínez, N., Ruiz, E., Galindo, R. y Galindo, L. (2015). La investigación acción en el trabajo colaborativo colegiado como estrategia para mejorar la práctica docente. Campus Virtuales, 4(1), 56-64. Recuperado de http://www.uaj ournals.com/campusvirtuales/es/ (Error 3: El enlace externo www.revistacampusvirtuales.es debe ser una URL) (Error 4: La URL www.revistacampusvirtuales.es no esta bien escrita)

Montero, L. (2011). El trabajo colaborativo del profesorado como oportunidad formativa. En Profesorado y calidad de la educación. Revista Participación Educativa, 16, 69-88. Recuperado de https://dialnet.unirioja.es/servlet/ articulo?codigo $=4942213$

Piña, J., Rodríguez, B. y Rodríguez, Y. (2016). Construcción del aprendizaje adulto. Revista ARJÉ, 10(18), 9-17. Recuperado de http://arje.bc.uc.edu.ve/arj18/art01.pdf

Pimienta, J. (2007). Metodología constructivista. Guía para la planeación docente. México: Editorial Pearson.

Quintero, C. y Romero, S. (2017). El colegiado docente en la formación de profesores. Revista de Investigación Educativa REDIECH, 7(13), 34-46. Recuperado de https://bit.ly/3rKgjT9

Revelo-Sánchez, O., Collazos-Ordoñez, C. y Jiménez-Toledo, J. (2018). El trabajo colaborativo como estrategia didáctica para la enseñanza/aprendizaje de la programación: una revisión sistemática de literatura. TecnoLógicas, 21(41), 115-134. Recuperado de http://www.scielo.org.co/pdf/teclo/v21n41/v21n41a08.pdf

Rodríguez, F. y Barraza, L. (2015). El trabajo colegiado y su influencia en la aplicación de estrategias de enseñanza. Durango, México: REDIE. Recuperado de http://www.redie.mx/librosyrevistas/libros/trabajocolegiado.pdf

Roselli, N. (2016). El aprendizaje colaborativo: Bases teóricas y estrategias aplicables a la enseñanza universitaria. Propósitos y Representaciones, 4(1), 219-280. Recuperado de https://revistas.usil.edu.pe/index.php/pyr/article/ view/90

Sandín, M. (2003). Investigación Cualitativa en Educación. Fundamentos y tradiciones. Madrid: Mc Graw- Hill.

Secretaría de Educación del Estado de Durango (SEED). (2019). Criterios para la elaboración de propuestas de Formación Continua y Superación Profesional. Durango, México: UFCySP/SEED.

Secretaría de Educación Pública (SEP). (1982). Manual de Organización del personal Adscrito al Subsistema de Educación Normal. México: Autor.

\section{Anexo 1.}

\section{Diseño curricular del Taller: Trabajo Colegiado y sus Implicaciones}

\section{PRESENTACIÓN}


El trabajo colegiado es una línea de política educativa que se ha planteado para las instituciones escolares, se pretende que a partir de la colaboración el profesorado pueda desarrollar estrategias que influyan en la mejora institucional. Sin embargo, en las Instituciones Formadoras de Docentes todavía se observan algunas dificultades en su interpretación, situación que redunda en escasa comprensión sobre las implicaciones de éste y que al final termina por ser un trabajo en equipo donde cada uno desde la individualidad plantea sus ideas, perdiendo así, la esencia de éste: el intercambio de ideas, la asunción de compromisos y el aprendizaje entre pares.

El taller tiene la finalidad de apoyar a profesores de la Escuela Normal en la resignificación del concepto trabajo colegiado y sus implicaciones, debido a que en un diagnóstico previo se detectó este problema, al hacer la valoración, se encontró que esto afecta en la toma de decisiones y en la asunción de compromisos porque existen concepciones diversificadas que dificultan la comunicación entre el colectivo docente y el desempeño en las aulas.

El taller consta de 15 horas, de éstas 10 son presenciales o bajo la conducción de un académico (BCA) y el resto son independientes (TI). Se dividió en tres unidades. Las que se desarrollarán en un periodo de dos sesiones de 5 hs (BCA) 2.5 hs (TI) cada una

\section{DEFINICIÓN DE LA OFERTA DE FORMACIÓN ACADÉMICA}

El taller está dirigido a docentes y directivos de la Escuela Normal.

\section{JUSTIFICACIÓN}

Este taller surge a iniciativa de un grupo de profesores de la Escuela Normal y se desprende de las actividades planeadas en el Diplomado: Investigación-Acción, cursado en el Instituto Educativo GUBA, capacitación contratada para sus docentes por los directivos de la misma institución, teniendo como objetivo desarrollar competencias investigativas usando como método la Investigación-Acción, puesto que las tareas y acciones que se desprenden del trabajo institucional y de práctica docente las demandan.

Para concretar esta propuesta de taller, el equipo de trabajo primeramente realizó un diagnóstico sobre el problema: ¿Qué está pasando con el trabajo colegiado en la Escuela Normal? Se aplicó una encuesta de respuesta abierta a 17 docentes de la escuela, donde se detectó una diversidad de conceptos y escasa comprensión sobre las implicaciones del mismo. Posteriormente se realizó revisión de literatura para reconocer estrategias de intervención y llegar así a la formulación del Plan de Acción.

Se optó por la modalidad de taller, debido a que permite la vinculación entre la teoría y la práctica, concentrando su énfasis en la última, requiere del dinamismo de los integrantes del grupo, es una metodología activa que exige la evidencia a través de los productos, donde la experiencia y el conocimiento previo son fundamentales para alcanzar nuevos saberes.

Se optó por la estrategia de aprendizaje colaborativo porque su finalidad es "Desarrollar personas reflexivas, autónomas y elocuentes" (Bruffee, 1995, en Barkley, Cross y Howell, 2012, p. 19). Además promueve el desacuerdo como punto para desequilibrar y equilibrar y es recomendada para la Educación superior.

\section{PROPÓSITO}

Promover en el personal docente de la Escuela Normal la resignificación de sus concepciones sobre el trabajo colegiado y sus implicaciones para favorecer una visión común en la toma de decisiones y el cumplimiento de acuerdos, mediante estrategias de aprendizaje colaborativo.

\section{PERFIL DE INGRESO}

- Capacidad para aprender por iniciativa e interés propio.

- Capacidad para comunicarse y expresar claramente sus ideas.

- Disposición para el trabajo colaborativo y respeto por la diversidad.

- Interés por participar con una conciencia ética en la vida institucional. PERFIL DE EGRESO

Identifica y comprende los elementos conceptuales del Trabajo Colegiado.

- Demuestra capacidad y disposición para realizar actividades que requieren del Trabajo Colegiado. 
- Conoce y tiene conciencia de las implicaciones del Trabajo Colegiado.

- Identifica semejanzas y diferencias entre el trabajo colaborativo, colegiado y cooperativo.

- Detecta la importancia de su participación en el desarrollo de las actividades institucionales.

- Toma decisiones de manera asertiva y responsable.

\section{COMPETENCIAS A FAVORECER}

- Comprende la importancia del trabajo colegiado y sus implicaciones en el desarrollo institucional, la toma de decisiones y la práctica docente para generar procesos de mejora que influyan en la formación integral del colectivo docente y los ambientes laboral y profesional, mediante el uso de estrategias apoyadas en el aprendizaje colaborativo.

- Genera propuestas de mejora que impacten al interior de la institución, por medio del trabajo colegiado, el aprendizaje colaborativo y compromiso con la Escuela Normal.

\section{CONTENIDOS}

Unidad 1 (Sesión uno, $2 \frac{1}{2}$ horas) (BCA)

- Generalidades del Trabajo: colaborativo, colegiado y cooperativo. Semejanzas y diferencias.

- Elementos conceptuales del Trabajo Colegiado.

Unidad 2 (Sesión uno, $2 \frac{1}{2}$ horas) (BCA)

- Implicaciones del Trabajo Colegiado en la Escuela Normal.

Unidad 3 (Sesión dos, 5 horas) (BCA)

- Actividades y estrategias que favorecen el Trabajo Colegiado.

- Aplicación y demostración de saberes.

ANEXO 1.

PROGRAMA CURRICULAR

\begin{tabular}{|c|c|c|c|c|c|}
\hline Unidades & No. sesiones & Contenidos & Mecanismos de evaluación & $\begin{array}{l}\text { Horas } \\
\text { Teóricas }\end{array}$ & $\begin{array}{l}\text { Horas } \\
\text { prácticas }\end{array}$ \\
\hline 1 & $1 / 2$ & $\begin{array}{l}\text { - Generalidades del } \\
\text { Trabajo: colaborativo, } \\
\text { colegiado y cooperativo. } \\
\text { Semejanzas y diferencias. } \\
\text { Elementos conceptuales del } \\
\text { Trabajo Colegiado. }\end{array}$ & $\begin{array}{l}\text { Evidencias de las } \\
\text { actividades desarrolladas } \\
\text { durante la sesión. } \\
\text { Participación individual y en } \\
\text { los equipos de trabajo. } \\
\text { Claridad y coherencia en las } \\
\text { exposiciones. }\end{array}$ & $2.5 \mathrm{hrs}$ & $2 \mathrm{hrs}$. \\
\hline 2 & $1 / 2$ & $\begin{array}{l}\text { - Implicaciones del Trabajo } \\
\text { Colegiado en la Escuela } \\
\text { Normal. }\end{array}$ & $\begin{array}{l}\text { - Vinculación entre el } \\
\text { conocimiento empirico y } \\
\text { teórico. }\end{array}$ & $2.5 \mathrm{hrs}$ & $2 \mathrm{hrs}$. \\
\hline 3 & 1 & $\begin{array}{l}\text { - Actividades y estrategias } \\
\text { que favorecen el Trabajo } \\
\text { Colegiado. Aplicación y } \\
\text { demostración de saberes. }\end{array}$ & $\begin{array}{l}\text { - Compromiso mostrado en } \\
\text { las actividades. Estrategias } \\
\text { presentadas para el uso del } \\
\text { trabajo colegiado. }\end{array}$ & $5 \mathrm{hrs}$. & 1 hra. \\
\hline
\end{tabular}

\section{CRONOGRAMA DE ACTIVIDADES}

Semana del 2 al 6 de marzo de 2020: organización del Taller

Semana del 7 al 11 de septiembre de 2020: primera y segunda intervención

Semana del 14 al 21 de septiembre de 2020: análisis de resultados

\section{METODOLOGÍA DE TRABAJO}

El enfoque del Taller es el Trabajo Colaborativo, con fundamento en técnicas de aprendizaje colaborativo de Barkley, Cross y Howell (2012). 
Asimismo, la estrategia del Taller, retoma planteamientos de Ander-Egg (1981), que destaca ocho aspectos que lo caracterizan:

a) Es un aprender haciendo. b) Es una metodología participativa. c) Es una pedagogía de la pregunta, contrapuesta a la pedagogía de la respuesta propia de la educación tradicional. d) Es un entrenamiento que tiende al trabajo interdisciplinario y al enfoque sistémico. e) La relación docente-alumno queda establecida en la realización de una tarea común. f) Carácter globalizante e integrador de su práctica pedagógica.g) Implica y exige de un trabajo grupal, y el uso de técnicas adecuadas. h) Permite integrar en un solo proceso tres instancias como son la docencia, la investigación y la práctica.

El Taller se llevará a cabo en la Escuela Normal en dos sesiones de 5 horas cada una; las sesiones se distribuirán en un periodo de cuatro semanas, considerando la organización institucional y disponibilidad de horario del personal docente convocado. Los participantes en el Taller serán principalmente quienes respondieron el cuestionario para el Diagnóstico, sin que represente una limitante para aquellos que deseen integrarse.

Se dará inicio con una dinámica de presentación y de integración, con la finalidad de que los participantes perciban un ambiente de confianza y de aprendizaje favorable; enseguida se dará a conocer el encuadre del Taller con apoyo visual en presentación de Power Point. Una vez que se aborda esta sección, se procede a retomar los elementos que se consideraron respecto a la concepción e implicaciones sobre el trabajo colegiado que percibe el colectivo docente.

Como actividades posteriores, se abordan semejanzas y diferencias del trabajo colaborativo, colegiado y cooperativo, además de elementos conceptuales básicamente del Trabajo Colegiado; esto se aborda de manera individual y enseguida en pequeños equipos para comparar y analizar planteamientos, que les permitan compartir al resto de los participantes.

Posteriormente se trabajará con el tema de las Implicaciones del Trabajo Colegiado, retomando la información previamente expuesta, teniendo claramente definidos los aspectos y elementos que lo conforman.

Reconocer las principales problemáticas, en la institución, asociadas al trabajo colegiado y sus implicaciones. A partir de ellas, buscar, proponer y enunciar estrategias y/o posibles alternativas de mejora; preparar una presentación, en power point $\mathrm{u}$ otro, mostrando la propuesta. De manera grupal, tomar acuerdos y asumir compromisos para lograr impacto positivo en la Escuela Normal. Redactar y compartir conclusiones.

La evaluación se realizará con base en los criterios que se plantean en el apartado de evaluación, tanto por cada sesión, como para cada producto y actividad que se desarrolle. En tanto que el taller será evaluado a partir de la aplicación de un instrumento diseñado para este fin.

\section{ESTRATEGIAS DIDÁCTICAS}

\section{SESIÓN UNO}

1. Exposición con apoyo en proyecciones de Power Point.

2. Análisis y discusión de ideas de manera grupal.

3. Lectura y revisión de textos de forma individual y por equipos.

4. Cuadro comparativo.

5. Mapa conceptual.

6. Presentación diversificada de la información.

7. Aprendizaje basado en construcción y reconstrucción de significados.

8. Mapa mental.

\section{SESIÓN DOS}

9. Presentación de la información.

10. Generar propuestas en equipo.

11. Puesta en común y reflexiones. 


\section{CRITERIOS DE EVALUACIÓN DE LOS PARTICIPANTES PROCESO}

- Demuestra disposición y capacidad para aprender por iniciativa e interés propio.

- Muestra capacidad y habilidad para comunicarse y expresar claramente sus ideas.

- Refleja disposición para el trabajo colaborativo en las actividades propuestas.

- Integración constante en las actividades a desarrollar en el taller.

- Identifica semejanzas y diferencias entre trabajo colegiado, colaborativo y cooperativo. PRODUCTO

- Presenta cuadro comparativo y mapa conceptual entre los tres tipos de trabajo abordados en las sesiones.

- Realiza mapa mental respecto a las implicaciones del Trabajo Colegiado.

- Elabora un escrito respecto al concepto e implicaciones del Trabajo Colegiado.

- Utiliza material de apoyo para exposición.

NIVEL DE LOGRO

- Logrado. Presenta en tiempo y forma trabajos individuales o en equipo; ejemplifica claramente las características planteadas; reflexiona las lecturas analizadas; realiza y entrega en tiempo y forma establecidos.

- En Proceso. Participa activamente en las actividades propuestas; tiene permanencia en el taller; genera propuestas de trabajo colegiado en la Escuela Normal; aporta ideas para las exposiciones a realizar; elabora y entrega aunque no en tiempo y forma.

- No Logrado. No participa activamente en las actividades propuestas, no asiste al $100 \%$ al taller y faltó elaborar y entregar los productos.

\section{PROCESO DE ACREDITACIÓN}

Para acreditar el programa y recibir su constancia, se deberá asistir al 100\% de las actividades presenciales y participar activamente en las actividades propuestas en el taller.

\section{MATERIALES DE TRABAJO}

Técnicas de aprendizaje Colaborativo (Barkley, et al., 2012, pp.17-31). El trabajo colegiado en las Instituciones Formadoras de Docentes (Barraza y Guzmán, 2007). El colegiado y sus realidades (Barraza y Barraza, 2014). Estrategias docentes para un aprendizaje significativo. Una interpretación constructivista (Díaz-Barriga y Hernández, 2002, pp. 99-127). El aprendizaje cooperativo en el aula (Johnson, Johnson y Hulobec, 1999). Metodología constructivista. Guía para la planeación docente (Pimienta, 2007). 


\section{Anexo 2.}

\section{Planeación Didáctica: Sesión 1}

ANEXO 2.

Planeación Didáctica: Sesión 1

\begin{tabular}{|c|c|c|}
\hline \multicolumn{3}{|c|}{ DATOS DE IDENTIFICACIÓN DE LA ESCUELA NORMAL } \\
\hline 1. CLASE NÚMERO: Sesión 1 & $\begin{array}{l}\text { 2. TEMA: Trabajo colegiado y sus } \\
\text { implicaciones }\end{array}$ & $\begin{array}{l}\text { 3. NIVEL DE } \\
\text { ASIMILACIÓN: Comprensión }\end{array}$ \\
\hline \multicolumn{3}{|c|}{$\begin{array}{l}\text { 4. OBJETIVO DEL APRENDIZAJE: Analizar el concepto de trabajo colegiado, mediante la comparación con el } \\
\text { trabajo cooperativo y colaborativo, para favorecer una visión común en la toma de decisiones y el cumplimiento de } \\
\text { acuerdos. OBJETIVO ACTITUDINAL: Tomar conciencia de sí mismo en el desempeño del trabajo colegiado. }\end{array}$} \\
\hline \multicolumn{3}{|c|}{ 5. TITULO DE LA CLASE: Significado del término. } \\
\hline 6. MÉTODO & $\begin{array}{l}\text { 7. ESTRATEGIA DE ENSEÑANZA- } \\
\text { APRENDIZAJE: }\end{array}$ & 8. RECURSOS \\
\hline Heuristico & $\begin{array}{l}\text { Cuadro comparativo Mapa conceptual } \\
\text { Mapa mental }\end{array}$ & $\begin{array}{l}\text { Computadora, proyector, } \\
\text { presentación en power point } \\
\text { sobre el diagnóstico, textos } \\
\text { sobre trabajo colegiado, } \\
\text { cooperativo y colaborativo } \\
\text { (incluidos en referencias). }\end{array}$ \\
\hline $\begin{array}{l}\text { 9. REACTIVACIÓN DE LOS } \\
\text { CONOCIMIENTOS PREVIOS }\end{array}$ & 10. SITUACIÓN PROBLEMÁTICA & $\begin{array}{l}\text { 13. APLICACIÓN DE LOS } \\
\text { CONOCIMIENTOS }\end{array}$ \\
\hline $\begin{array}{l}\text { ¿Qué entiendes por trabajo colegiado? } \\
\text { ¿Qué entiendes por trabajo } \\
\text { cooperativo? ¿Qué entiendes por } \\
\text { trabajo colaborativo? }\end{array}$ & $\begin{array}{l}\text { Se mostrará el análisis de las } \\
\text { concepciones sobre trabajo colegiado } \\
\text { recuperadas del diagnóstico, con la } \\
\text { finalidad de que el colectivo docente } \\
\text { visualice la diversidad de conceptos que } \\
\text { se asumen. }\end{array}$ & $\begin{array}{l}\text { En colegiado, construyen un } \\
\text { concepto unificado de trabajo } \\
\text { colegiado. }\end{array}$ \\
\hline $\begin{array}{l}\text { 11. CONSTRUCCIÓN DE } \\
\text { SIGNIFICADOS }\end{array}$ & $\begin{array}{l}\text { 12. ORGANIZACIÓN DEL } \\
\text { CONOCIMIENTO }\end{array}$ & $\begin{array}{l}\text { 14. EVALUACIÓN DEL } \\
\text { PROCESO }\end{array}$ \\
\hline \multirow{3}{*}{$\begin{array}{l}\text { Presentar un mapa conceptual sobre } \\
\text { los tipos de trabajo para que el } \\
\text { profesorado haga su interpretación. } \\
\text { Contrastar con los textos para validar } \\
\text { sus aprendizajes. }\end{array}$} & \multirow[b]{3}{*}{ Elabora un cuadro comparativo } & $\begin{array}{l}\mathrm{S} \text { ¿Qué sé? Q ¿Qué quiero } \\
\text { aprender? A ¿Qué aprendi? }\end{array}$ \\
\hline & & 15. TAREA \\
\hline & & $\begin{array}{l}\text { Un escrito breve sobre los } \\
\text { aprendizajes nuevos que se } \\
\text { obtuvieron sobre el trabajo } \\
\text { colegiado y sus implicaciones }\end{array}$ \\
\hline \multicolumn{3}{|c|}{$\begin{array}{l}\text { MATERIALES DE TRABAJO El colegiado y sus realidades (Barraza y Barraza, 2014). Estrategias docentes para un } \\
\text { aprendizaje significativo (Diaz-Barriga y Hernández, 2002, pp. 83-114). Aprendizaje Colaborativo en la Formación } \\
\text { Universitaria de Pregrado (Lillo, 2013, pp. 109-142). Técnicas de aprendizaje Colaborativo (Barkley, et al., 2012, pp. } \\
\text { 17-31). }\end{array}$} \\
\hline
\end{tabular}

Fuente: Formato construido a partir de Pimienta (2007). 


\section{Anexo 3.}

\section{Planeación Didáctica: Sesión 2}

ANEXO 3.

Planeación Didáctica: Sesión 2

\begin{tabular}{|c|c|c|}
\hline \multicolumn{3}{|c|}{ DATOS DE IDENTIFICACIÓN DE LA ESCUELA NORMAL } \\
\hline 1. CLASE NÚMERO: Sesión 2 & $\begin{array}{l}\text { 2. TEMA: Estrategias que favorecen el } \\
\text { Trabajo colegiado }\end{array}$ & $\begin{array}{l}\text { 3. NIVEL DE ASIMILACIÓN: } \\
\text { Aplicación }\end{array}$ \\
\hline \multicolumn{3}{|c|}{$\begin{array}{l}\text { 4. OBJETIVO DEL APRENDIZAJE: Generar estrategias que contribuyan al desarrollo del trabajo colegiado en la } \\
\text { Escuela Normal. OBJETIVO ACTITUDINAL: Contribuir al desarrollo del trabajo colegiado. }\end{array}$} \\
\hline \multicolumn{3}{|c|}{ 5. TITULO DE LA CLASE: Aplicación y demostración de saberes. } \\
\hline 6. MÉTODO & $\begin{array}{l}\text { 7. ESTRATEGIA DE ENSEÑANZA- } \\
\text { APRENDIZAJE: }\end{array}$ & 8. RECURSOS \\
\hline Heuristico & Preguntas exploratorias & $\begin{array}{l}\text { Computadora, proyector, textos } \\
\text { sobre trabajo colegiado, } \\
\text { cooperativo y colaborativo } \\
\text { (incluidos en referencias). }\end{array}$ \\
\hline $\begin{array}{l}\text { 9. REACTIVACIÓN DE LOS } \\
\text { CONOCIMIENTOS PREVIOS }\end{array}$ & 10. SITUACIÓN PROBLEMÁTICA & $\begin{array}{l}\text { 13. APLICACIÓN DE LOS } \\
\text { CONOCIMIENTOS }\end{array}$ \\
\hline $\begin{array}{l}\text { Mediante la estrategia PNI, recuperar } \\
\text { ideas del profesorado respecto a los } \\
\text { aspectos positivos, negativos e } \\
\text { interesantes del trabajo colegiado. }\end{array}$ & $\begin{array}{l}\text { ¿Qué implicaciones tiene el Trabajo } \\
\text { Colegiado para el colectivo docente de } \\
\text { la Escuela Normal? }\end{array}$ & $\begin{array}{l}\text { Planear una sesión de trabajo } \\
\text { colegiado, a partir de los } \\
\text { formatos e indicadores } \\
\text { propuestos como sugerencia. }\end{array}$ \\
\hline $\begin{array}{l}\text { 11. CONSTRUCCIÓN DE } \\
\text { SIGNIFICADOS }\end{array}$ & $\begin{array}{l}\text { 12. ORGANIZACIÓN DEL } \\
\text { CONOCIMIENTO }\end{array}$ & $\begin{array}{l}\text { 14. EVALUACIÓN DEL } \\
\text { PROCESO }\end{array}$ \\
\hline \multirow[t]{3}{*}{$\begin{array}{l}\text { Proponer una actividad dinámica que } \\
\text { implique el trabajo colegiado. }\end{array}$} & \multirow[t]{3}{*}{$\begin{array}{l}\text { Proporcionar diferentes esquemas para } \\
\text { organizar el trabajo colegiado en la } \\
\text { Escuela Normal. }\end{array}$} & $\begin{array}{l}\text { Valoración positiva -negativa del } \\
\text { trabajo colegiado. } \\
\text { Reconocimiento de implicaciones } \\
\text { individuales, colectivas e } \\
\text { institucionales para el desarrollo } \\
\text { del trabajo colegiado. Diseño de } \\
\text { estrategias para aplicar el trabajo } \\
\text { colegiado. Pertinencia de las } \\
\text { propuestas para desarrollar } \\
\text { trabajo colegiado }\end{array}$ \\
\hline & & 15. TAREA \\
\hline & & $\begin{array}{l}\text { Propuesta colectiva para } \\
\text { desarrollar trabajo colegiado. }\end{array}$ \\
\hline
\end{tabular}

Fuente: Formato construido a partir de Pimienta (2007).

\section{INFORMACIÓN ADICIONAL}

Cómo citar: Aguirre Gómez, F.J. y Barraza Barraza, L. (2021). El trabajo colegiado y sus implicaciones: diseño de una propuesta pedagógica. Revista Educación, 45(2). Recuperado de http://doi.org/10.15517/revedu.v4 5i1.42985 